\title{
Impact of marijuana smoking on lung function in older persons
}

\author{
To the Editor:
}

The investigation by TAN et al. [1] addresses an important question regarding the impact of marijuana smoking in middle-aged and older individuals and the relative risk for developing COPD and an accelerated rate of decline in lung function. The importance of this question is underscored by the spreading legalisation of marijuana and the likely impact on its use in both older and younger persons. The authors approached this question using data from a Canadian cross-sectional population-based prevalence study of COPD carried out in subjects $\geqslant 40$ years (the COLD cohort) and a selected subsample who were followed for up to 4 years and either had physiological evidence of COPD (forced expiratory volume in $1 \mathrm{~s}\left(\mathrm{FEV}_{1}\right)$ to forced vital capacity ratio $\left.<0.70\right)$ or served as age- and gender-matched control never and ever smokers with normal lung function (CanCOLD cohort). Since the vast majority (83\%) of marijuana smokers in their study also smoked tobacco, the analysis was carried out by adjusting for the concomitant use of marijuana in the dual smokers. Dose-response was analysed by categorising the smokers using three levels of cumulative life-time exposure defined by the number of pack- or joint-years (mild, 1-5; moderate, >5-20; and heavy, >20), and the level of exposure, along with other influential covariates, was adjusted for in the analyses. In their cross-sectional study the authors found that heavy marijuana smoking ( $>20$ joint-years) was associated with spirometrically defined COPD, while in their longitudinal study heavy marijuana smokers were found to experience a substantially faster annual rate of $\mathrm{FEV}_{1}$ decline (nearly $30 \mathrm{~mL} \cdot \mathrm{yr}^{-1}$ compared to never smokers of either substance) that was even faster than the decline observed in the heavy tobacco smokers (slightly more than $20 \mathrm{~mL} \cdot \mathrm{yr}^{-1}$ ).

Based on their findings, the authors concluded that heavy marijuana smoking was independently associated with chronic airflow obstruction and is a risk factor for progression of airways disease. However, the study suffers from a number of important limitations, many of which are pointed out in the accompanying editorial by HANCOX and SEARS [2], leading one to question the validity of these conclusions. An additional concern is the manner in which the authors selected the study sample. Of the 5291 participants in the original cross-sectional study, the prospective longitudinal CanCOLD cohort included only 1285 subjects. The CanCOLD cohort purposely focused on tobacco and marijuana smokers with COPD, as determined from their baseline spirometry, while never smokers with normal lung function were used as controls. In this setting it is no surprise that smokers of either or both substances would exhibit greater rates of decline in lung function than never smokers given that the former were identified as having COPD, in whom an accelerated rate of decline is characteristic, while the latter were not. In other words, the design of the CanCOLD sample biased toward showing a greater rate of decline in $\mathrm{FEV}_{1}$ among marijuana smokers, many of whom were enrolled based on prior evidence of COPD.

Another important issue relates to the very small number of marijuana-only smokers in the longitudinal cohort ( $\mathrm{n}=33$ at visit 1,23 at visit 2 and 18 at visit 3 , of whom only three were heavy marijuana smokers). Since the number of heavy marijuana-only smokers is so low, the findings in relation to heavy marijuana smokers are mainly based on dual smokers of both marijuana and tobacco. While the authors indicate that they adjusted for concomitant tobacco smoking in their analysis, one might reasonably question whether such adjustment truly gets at the truth regarding the independent effects of heavy marijuana smoking, particularly when so very few subjects were heavy smokers of marijuana only.

An additional concern has to do with the unexpected finding that the adjusted annual rate of $\mathrm{FEV}_{1}$ decline in the former heavy marijuana smokers was very close to that of the current heavy marijuana smokers (figure 3a), a finding that is at variance with the results for the former tobacco smokers, whose annual rate

@ERSpublications

The report by W.C. Tan and co-workers raises important questions regarding the impact of marijuana smoking on lung function in older individuals but fails to directly answer them http://bit.ly/2LP2AYi

Cite this article as: Tashkin DP, Roth MD. Impact of marijuana smoking on lung function in older persons. Eur Respir J 2020; 55: 1902328 [https://doi.org/10.1183/13993003.02328-2019]. 
of decline is considerably less than that for the current tobacco smokers (figure $3 \mathrm{~b}$ ), consistent with the previously described finding that sustained cessation of tobacco smoking leads to a nearly complete reversal of the accelerated annual rate of decline of $\mathrm{FEV}_{1}$ in individuals with COPD toward the age-related decline noted in never smokers [3]. Since most of the marijuana smokers in the current study also smoked tobacco, one wonders whether these paradoxical findings might reflect the likelihood that dual smokers of marijuana and tobacco will often cease smoking marijuana while continuing to smoke tobacco, a more addictive substance, as has been reported previously [4], so that the continuingly rapid decline in lung function in former marijuana smokers might simply reflect the effect of continued tobacco smoking.

Given these important methodological and analytical issues, as well as the other limitations noted in the accompanying editorial [2], this study raises an important questions regarding the potential impact of marijuana use on the rate and progression of COPD in older smokers of marijuana, and its interaction with tobacco smoking, but it does not directly answer them. In addition to raising important questions, this study points out the need for prospective studies designed specifically to assess the long-term effects of inhaled marijuana smoke on lung function change over time in an older population. An example would be a population-based study of a random sample of middle-aged and older smokers and nonsmokers not selected on the basis of prior evidence of COPD. Marijuana smokers, healthcare professionals and the public at large deserve direct and well-founded answers to the questions posed by TAN et al. [1].

Donald P. Tashkin and Michael D. Roth

Dept of Medicine, David Geffen School of Medicine at UCLA, Los Angeles, CA, USA.

Correspondence: Donald P. Tashkin, Dept of Medicine, David Geffen School of Medicine at UCLA, 10833 Le Conte Ave, Los Angeles, CA 90095, USA. E-mail: dtashkin@mednet.ucla.edu

Received: 5 Dec 2019 | Accepted: 7 Dec 2019

Conflict of interest: D.P. Tashkin has nothing to disclose. M.D. Roth has nothing to disclose.

\section{References}

1 Tan WC, Bourbeau J, Aaron SD, et al. The effects of marijuana smoking on lung function in older people. Eur Respir J 2019; 54: 1900826.

2 Hancox RJ, Sears MR. The impact of marijuana smoking on lung function. Eur Respir J 2019; $54: 1902065$.

3 Scanlon PD, Connett JE, Waller LA, et al. Smoking cessation and lung function in mild-to-moderate chronic obstructive pulmonary disease: The Lung Health Study. Am J Respir Crit Care Med 2000; 161: 381-390.

4 Simmons MS, Tashkin DP. The relationship of tobacco and marijuana smoking characteristics. Life Sci 1995; 56: 2185-2191.

From the authors:

Most previous epidemiological studies support a significant association between marijuana smoking and chronic respiratory symptoms [1], but the effects on lung function remain unclear. Previous longitudinal cohort studies have focused largely on young adults [1]. The results from these studies have shown that marijuana smoking produced marginal or no effects on lung function, which is expected given that with even heavy cigarette exposure, meaningful changes in forced expiratory volume in $1 \mathrm{~s}\left(\mathrm{FEV}_{1}\right)$ or forced vital capacity (FVC) are hard to discern in young adults. Lung function changes, however, accelerate in older age groups and the impact of respiratory irritants and toxins on lung function becomes much more evident [2]. To our knowledge, our study is the first longitudinal study of marijuana smoking in older individuals in a general population whose median age was 65 years [3]. The results of the present study addressed a major gap in marijuana research by demonstrating that marijuana smoking amplifies the harmful effects of tobacco smoking on the risk of COPD and $\mathrm{FEV}_{1}$ decline over time. 
D.P. Tashkin and M.D. Roth expressed concerns regarding the conclusion of the study because of the limitations of the design. Most of these issues had been systematically described in the discussion section of the published paper [3]. We agree with the authors that future large longitudinal studies of sole marijuana smoking in the general population are urgently needed. In the meantime, our results elaborate on some critical gaps in knowledge, and can inform public policy debate and guide research direction on the effects of smoked marijuana in certain clinical contexts.

We also agree with D.P. Tashkin and M.D. Roth that the sample size for the subgroup of marijuana only smokers was very small. Accordingly, in the discussion section, we de-emphasised the potential "independent" relationship between marijuana smoking and poor respiratory outcomes, and cautioned the readers against over-interpretation of these data. In contradistinction, the sample size in the tobacco plus marijuana group was large and robust. In this subgroup, we found that marijuana smoke exposure amplified the harmful effects of cigarette smoke on various respiratory end-points, including the risk of COPD (figure 1a and b), and decline in $\mathrm{FEV}_{1}$ (tables 3 and 4) and in FVC (supplementary table E4). Importantly, for the two key latter end-points, we demonstrated significant interaction between tobacco smoke and marijuana smoke ( $\mathrm{p}=0.042$ for interaction between marijuana and tobacco smoking for $\mathrm{FEV}_{1} /$ $\mathrm{FVC}$ ratio and $\mathrm{p}<0.00001$ interaction for $\mathrm{FEV}_{1}$ decline), indicating a high level of synergy for harm.

Our choice of the "design of the CanCOLD sample" was deliberate: our intent was to determine, in a cohort of smoking subjects with spirometrically proven COPD, who were clearly at risk of accelerated declines in lung function, whether concomitant marijuana and tobacco smoking proved to increase the risk of an accelerated decline in lung function relative to those who only smoked tobacco. Our choice of this high-risk cohort enabled us to discover significant interaction effects suggesting that marijuana smoke exposure amplified the harmful effects of cigarette smoke exposure on risk of decline in $\mathrm{FEV}_{1}$ over time.

Another issue raised by D.P. Tashkin and M.D. Roth is the age difference between tobacco-only smokers and dual smokers. On average, the smokers of tobacco only were 12 years older than the marijuana plus tobacco smoker group, likely reflecting the differences in attitude regarding marijuana smoking across the age spectrum. While we agree that differential age distribution may lead to confounding, we used well-accepted statistical approaches to adjust for these differences. Furthermore, because lung function declines accelerate with age, any residual effects caused by differential ageing between the two groups would have led to an underestimation of the true impact of marijuana smoking on lung function. Thus, our findings demonstrating the harmful effects of smoked marijuana in this dual smoking group may have been conservative.

We would like to clarify the issue of dropout rate, as mentioned by HANCOX and SEARS [4] in their commentary. In this prospective, non-interventional, cohort, individuals varied in the number of repeated measurements they contributed and at the time at which these were obtained, due to participant scheduling availabilities and preferences. The actual retention rate (i.e. number of people still in the study) was $81 \%$ in 2017 , when the data were censored and used for analysis in the manuscript.

The fact that most long-term marijuana smokers also smoked tobacco in this population-derived cohort is a reflection of the real-world pattern of marijuana smoking in the USA and Europe to date [5, 6]. Perhaps, with increasing societal acceptance of smoked marijuana and its use in the population, future studies will be able to evaluate the effects of smoked marijuana in isolation on the risk of COPD and its progression. Until then, our findings indicate caution in the use of smoked marijuana, especially in those who also smoke cigarettes. We believe that evidence and not a priori beliefs or zeal should inform public discourse on merits of marijuana availability and use in the population [7]. To this end, we welcome the commentary by HaNCOX and SEARs [4] and the correspondence from D.P. Tashkin and M.D. Roth.

Wan C. $\operatorname{Tan}^{1}$, Jean Bourbeau $\oplus^{2}$, Shawn D. Aaron ${ }^{3}$ and Don D. $\operatorname{Sin}^{1}$, on behalf of the CanCOLD Collaborative Research Group

${ }^{1}$ The University of British Columbia, Center for Heart Lung Innovation, St Paul's Hospital, Vancouver, BC, Canada. ${ }^{2}$ Research Institute McGill University Health Center, McGill University, Montreal, QC, Canada. ${ }^{3}$ The Ottawa Hospital Research Institute, University of Ottawa, Ottawa, ON, Canada.

Correspondence: Wan C. Tan, University of British Columbia, Centre for Heart Lung Innovation, St Paul's Hospital, Rm166, 1081 Burrard Street, Vancouver, BC V6Z 1Y6, Canada. E-mail: wan.tan@hli.ubc.ca

Received: 12 Dec 2019 | Accepted: 13 Dec 2019

Conflict of interest: W.C. Tan reports grants from Canadian Institute of Heath Research (CIHR/R\&D Collaborative Research Program Operating Grants 93326) with industry partners AstraZeneca Canada Ltd, Boehringer Ingelheim Canada Ltd, GlaxoSmithKline Canada Ltd, Merck, Novartis Pharma Canada Inc., Nycomed Canada Inc., Pfizer Canada Ltd, during the conduct of the study. J. Bourbeau reports grants from Canadian Institute of Heath Research (CIHR/ R\&D Collaborative Research Program Operating Grants 93326) with industry partners AstraZeneca Canada Ltd, 
Boehringer Ingelheim Canada Ltd, GlaxoSmithKline Canada Ltd, Merck, Novartis Pharma Canada Inc., Nycomed Canada Inc., Pfizer Canada Ltd, during the conduct of the study. S.D. Aaron has nothing to disclose. D.D. Sin reports grants from Merck, personal fees for advisory work from Sanofi-Aventis and Regeneron, grants and personal fees from Boehringer Ingelheim, grants and personal fees for lectures and advisory board work from AstraZeneca, personal fees for lectures and advisory board work from Novartis, outside the submitted work.

\section{References}

1 Ghasemiesfe M, Ravi D, Vali M, et al. Marijuana use, respiratory symptoms, and pulmonary function: a systematic review and meta-analysis. Ann Intern Med 2018; 169: 106-115.

2 Gan WQ, Man SF, Postma DS, et al. Female smokers beyond the perimenopausal period are at increased risk of chronic obstructive pulmonary disease: a systematic review and meta-analysis. Respir Res 2006; 7: 52.

3 Tan WC, Bourbeau J, Aaron SD, et al. The effects of marijuana smoking on lung function in older people. Eur Respir J 2019; 54: 1900826.

4 Hancox RJ, Sears MR. The impact of marijuana smoking on lung function. Eur Respir J 2019; 54 : 1902065.

5 Agrawal A, Budney AJ, Lynskey MT. The co-occurring use and misuse of cannabis and tobacco: a review. Addiction 2012; 107: 1221-1233.

6 Hernandez-Serrano O, Gras ME, Font-Mayolas S. Concurrent and simultaneous use of cannabis and tobacco and its relationship with academic achievement amongst university students. Behav Sci (Basel) 2018; 8: E31.

7 Tan WC, Sin DD. What are the long-term effects of smoked marijuana on lung health? CMAJ 2018; 190: E1243-E1244. 